\title{
ORAL CANCER ITS ETIOLOGY \& CONCERNS: A REVIEW
}

\section{Anitha $\mathrm{S}^{1}$}

\section{HOW TO CITE THIS ARTICLE:}

Anitha S. "Oral Cancer Its Etiology \& Concerns: A Review". Journal of Evolution of Medical and Dental Sciences 2015; Vol. 4, Issue 55, July 09; Page: 9660-9664, DOI: 10.14260/jemds/2015/1393

ABSTRACT: Oral cancer is the largest group of cancer that categorizes into the head and neck region and worldwide health problem. Exact cause of oral cancer is unknown. Risk factors for cancer could be varied including Iron deficiency anemia, are alcohol, tobacco, immunologic susceptibility, gene mutations, epithelial cell growth, suppressor proteins and disease of chemotherapy, such as in cases of lymphoma and leukemia. Role of viruses have also been reported in the etiology. Dietary factors such as high fat and low fiber may play a role in carcinogenesis in some sites. High incidence of alcoholism is relevant because alcohol intake has been related to an increase risk of developing oral cancer. Other signaling factors have also been proposed to have a role. The paper presents a review on oral cancer, its causes \& concern.

KEYWORDS: oral cancer, signaling factors in oral cancer, smoking, viruses, risk factors of oral cancer

INTRODUCTION: Neoplasms are autonomous new growths. They arise spontaneously and their growth is not limited by the biological restraints that control the growth of other cells. They always produce deleterious effect and cause death of host. Neoplasms are basically divided into benign and malignant. ${ }^{1}$ The term "oral cancer" is used to describe any malignancy that arises from oral tissues. ${ }^{2}$ Oral cancer is the largest group of cancer that categorizes into the head and neck region. It is a worldwide health problem with new cases exceeding 640000. Oral cancer is a major health problem in India, with $10 \%$ of estimated 644,600 new cancers that occur in all parts of the body each year. On the basis of annual age adjusted incidence rate, oral cancer rank $1^{\text {st }}$ to 6 thamong all cancers in India. Generally oral cancer occurs more commonly among men than women depending on the extent and type of tobacco habit with the highest number occurring in the 6th decade. According to WHO, 90\% of oral cancer in south East Asia is attributed to tobacco use. ${ }^{3}$

Early detection of mouth cancer (i.e., when lesions are less than $3 \mathrm{~cm}$ in size and show no evidence of deep invasion and metastasis) dramatically increases the survival rate. Unfortunately over the past few decades there has been only small improvement in the early diagnosis of oral cancer, over $50 \%$ of all cancer cases are extensive, late stage malignancies at the time of diagnosis/Hence treatment is aggressive which increases morbidity and result only in slight improvement in survival rate. Therefore early diagnosis of a lesion during the localized stage combined with adequate treatment appears to be the most effective way to further improves oral cancer control. ${ }^{4}$ Of the total oral cancer, carcinomas account for $96 \%$ and sarcoma $4 \% .5$ Oral tongue squamous cell carcinoma is one of the most common sites of head and neck cancer. Its normal epidemiology shows a persistent trend of an increase in incidence among young people in some regions of the world. ${ }^{6}$

Etiology and Predisposing Factor: Exact cause of oral cancer is unknown. Variation in incidence rate among different group or population may be influenced by difference in exposure to carcinogenic initiator or promoter. The various risk factors for cancer are alcohol, tobacco, immunologic susceptibility, gene mutations,, 46 epithelial cell growth, suppressor proteins and disease 
of chemotherapy, such as in cases of lymphoma and leukemia.6,7 The oral cavity may be one of the first sites of signs and symptoms that lead to diagnosis of these diseases. For persons in general population who develop oral cancer and who are not otherwise known to have or be at risk of immunodeficiency it is not clear whether the difference found in some immunologically variant cause or result from the malignancy.

Carcinoma-Associated Fibroblast (CAF): is the most important host cell type in tumor microenvironment, which greatly contributes to tumor initiation, progression, and metastasis. Therefore, a large amount of data has emerged, showing the cancer-promoting function of these cells via paracrine effects that escort tumor cells through all the steps of cancer development. CAF is a heterogeneous cell population that can arise from the differentiation of resting fibroblasts, epithelial cells, endothelial cells, and mesenchymal stem cells. This review summarizes the current knowledge of the role of CAFs in tumor progression, with a particular focus on the cellular and molecular features and recent advances in researches on the genetic status and microRNA regulation, and addresses the potential prognostic and therapeutic values for patients with oral cancer by targeting CAFs. ${ }^{4,6}$

Tobacco: Smoking may be viewed as a worldwide endemic causing serious disease and immense health problems apart from its effect of mortality smoking results in increased morbidity rate ${ }^{9}$. Tobacco is addictive and is known to be harmful to health in many ways. Both smoked and smokeless tobacco contains alkaloids, nicotine which is the main addictive agents. They as well contain thousands of chemical compounds, which are not only irritants and toxins but also carcinogen. Most specific carcinogen is tobacco specific nitrosamine polycyclic aromatic hydrocarbons and many others. ${ }^{3,7}$

Tobacco Habit in India: Tobacco habits are practiced in various different forms many of them are specific to certain areas of India. The reasons for the initiation of tobacco are many. Generally the important reason is a tooth related complaint (48.1\%), followed by peer group influence (38\%), tooth related problem were the most common reason for women $(92.1 \%)$, where as for men peer group influence (58.1\%) was more important. ${ }^{7}$

Oral Cancer Risk and Smoking: The association between cigarette used and oral carcinoma has been firmly established from epidemiologic studies revealing that there are more than twice as many smokers among oral cancer patients as among control population. A study of 403 oral and pharyngeal cancer patients followed for a mean of 5.1 years at the University of California San Fransisco found that $72.1 \%$ were smokers and $58.1 \%$ smoked more than 1 pack per day. Demonstrating the very high risk to tobacco users in the follow up study it was found that almost 1 of the 5 patients (17.7\%) developed second primary oral and oro-pharyngeal cancer in a mean time of 5 years. smoked tobacco are in the following forms-ciagarette, bidi, cigar/chutta, reverse chutta, dhunti, reverse dhunti, hookli, chilum, hooka. Smokeless tobacco is in the following form pan, pan masala, main puri, mava, and tobacco lime preparation. 3,8

Alcohol: High incidence of alcoholism is relevant because alcohol intake has been related to an increase risk of developing oral cancer and a higher than an expected mortality. More than 30 years ago study determined that heavy alcohol consumption was a significant factor in the development of 
mouth cancer .In this study one-third of the 543 male patients drunk more than 7 ounce of whisky per day as compared with $12 \%$ of control group. One group of investigators found that $44 \%$ of 108 patients with cancer of the tongue and 59\% of 68 patients with cancer of floor of mouth, palate, tonsilar fossa had unequivocal evidence of alcoholic cirrhosis. Keller in a study concluded that cirrhosis; heavy drinking and smoking were associated with cancer. ${ }^{9}$ Most heavy drinkers are also smokers and their predisposing factors probably work in combination rather than independently. ${ }^{10}$

Oral Lichen Planus: Is a complex, chronic inflammatory disease. Mucosa \& skin both may be affected. Buccal mucosa is the most common location. Reports have associated OLP with malignant transformation. In a report from UK, review of 241 OLP patients found that $37 \%$ developed a carcinoma transformation up to 12 years. In this review of studies performed since 1981, the author reported oral lichen planus associated malignancies varying $0.4 \%$ to $5.6 \%$ in 7 different countries. ${ }^{11}$

Denture Wearing: Various studies show no difference between denture wearers and control group in the occurrence of oral cancer.7,11

Nutrition: Dietary factors such as high fat and low fiber may play a role in carcinogenesis in some sites. Deficiencies of antioxidant, vitamins and nutrients have been postulated as co-factors in carcinogenesis, more specifically cancer of mouth, larynx and esophagus have been related to the low intake of fruits and vegetables. Active ingredients in fruits and vegetables that may act as suppressor agents include indoles, flavonoids, isothiocynates, therpenes, rutin and phenolic antioxidants. Low dietary and/ or serum levels of vitamin A have been associated with oral precancerous lesions and subsequent cancer. This association has been based upon the co-relation between vitamin A deficiencies and hyperkeratosis, beta-carotene, a precursor to vitamin A has not been effective in the control of pre-malignant or cancerous oral lesion. ${ }^{12}$ In a recent study, it was found that consumption of fruits and vegetables is associated with a reduced risk of oral cancer..$^{13}$

Iron deficiency anemia is part of the Plummer-Vinson syndrome, which has been associated with an increased risk of developing carcinoma of tongue. Iron deficiency has not been shown to be a common finding in patients presenting with oral cancer, although high Iron storage has been indicated as a possible marker or factor in cancer, carcinoma of tongue. Iron deficiency has not been shown to be a common finding in patients presenting with oral cancer, although high Iron storage has been indicated as a possible marker or factor in cancer.11,12,13,14

Virus: The role of viruses in the etiology of oral cancer has been matter of speculation for a long time. Despite years of clinical observation and research, the exact role of viruses is still rather speculative. ${ }^{15,16}$

Herpes Virus: There are 8 types of herpes simplex virus that infect human tissue. Herpes simplex virus type 1(HSV1), herpes simplex virus type 2(HSV2), Varicella zoster virus (VZV), Human cytomegalovirus (CMV), Epsteinbarr virus (EBV), Human herpes virus 6, 7, 8(HHV-6, HHV-7, HHV-8):

a) Herpes simplex virus type 1: HSV1 is well known cause of primary herpes stomatitis and recurrent herpes labialis. Although the virus is highly cytolytic and destroys infected cells efficiently, it does in fact have the ability to transfer from cells to a malignant phenotype under some circumstances. 
b) Epstein barr virus-EBV is almost certainly an etiologic agent of Burkett lymphoma in Africa and is also closely associated with nasopharyngeal carcinoma in several Asian countries. EBV is present in Hairy Leukoplakia in all parts of the world. Since an oral cancer does not appear to arise in hairy leukoplakia, it seems doubtful that EBV plays any role in development of oral cancer.

c) Human herpers virus 6: HHV6 originally developed in patients with acquired immuno deficiency syndrome and might play a role in the disease, since it can increase expression of several functions of HIV.

d) Human herpes virus 8: This is the most recently discovered human herpes virus found in lesions of AIDS associated Kaposi's sarcoma.

e) Papilloma virus: more than 70 papilloma viruses have been identified. The principle high risk of human papilloma virus is type 16 and type ${ }^{9,17}$

\section{OTHER CAUSES ATTRIBUTED TO CAUSES OF CANCER ARE:}

Repression of $G$ protein coupled receptor family C group 5 member A associated with pathologic differentiation grade of oral squamous cell carcinoma. 18

TGF-beta1, Smad-2/3, Smad-1/-5/=8 and Smad-4 signaling factors are expressed in ameloblastomas, adenomatoid odontogenic tumor, and calcifying cystic odontogenic tumor.

Expression of human epidermal growth factor receptor (HER), especially EGFR and HER4 in odontogenic tissue suggesting that growth signals mediated by these receptor molecules contribute to cell proliferation, survival and differentiation in both normal and neoplastic odontogenic epithelial tissues. ${ }^{18}$

Syndecan-1(SD 138) expression by the tumors stroma is considered to be associated with poor prognosis of ameloblastoma, KCOT, and dentigerous cyst. 19

Expression of Prox-1 in the neoplastic spindle cells supports the lymphatic differentiation in oral kaposi's sarcoma. ${ }^{19}$

Elevated level of serum growth differentiation factor 15 is associated with oral leukoplakia and oral squamous cell carcinoma. ${ }^{19}$

CONCLUSION: Oral cancer has myriad clinical presentation. Identifying its etiology, adequate diagnosing skills \& appropriate interventions are required.

\section{REFERENCES:}

1. Al-Otaibi 0 et al. Syndecan-1 (CD 138) surface expression marks cell type\& differentiation in ameloblastoma, keratocystic odontogenic tumor, and dentigerous cyst. J Oral Pathol \& medicine (2013) 42:186-193.

2. Colby, Kerr and Robinson's, Color atlas of oral pathology forth edition, page no 137.

3. Fali S Mehta, James E Hamner, A lesion with serious outcome, oral cancer, tobacco related oral mucosal lesions and conditions in India, page 1. 
4. Jingyi $\mathrm{W}$ et al. Genetic regulation and potentially therapeutic application of cancer-associated fibroblasts in oral cancer. J Oral Pathol Med 2014 43:323-334.

5. Oral cancer, $4^{\text {th }}$ edition, Sol Silverman, Publisher. B. C. decker Inc; p7.

6. Meir Gorsky DMD, Sol Silverman Jr MA. Denture wearing and oral cancer, Journal of prosthetic dentistry, Aug 1984,Vol 52(2): 164-166

7. Oral cancer, $4^{\text {th }}$ ed, Sol Silverman, Publisher. B. C. decker Inc; p14.

8. Liu S. Et al. 'Repression of G protein-coupled receptor family $\mathrm{C}$ group 5 member $\mathrm{A}$ is associated with pathologic differentiation grade of oral squamous cell carcinoma. J Oral Pathol \& medicine (2013) 42:761-768.

9. Oral cancer, $4^{\text {th }}$ ed, Sol Silverman, Publisher. B. C. decker Inc; p20-21.

10. Shang- Yang Yu et al. increased expression of MCM5 is significantly associated with aggressive progression and poor prognosis of oral squamous cell carcinomaJ Oral Pathol Med 2014 43:344-349.

11. Stevens RG et al body iron stores and risk of cancer. N Engl J Med 1988; 319:1047-1055.

12. Pavia $M$ et al. Association between fruits and vegetables consumption and oral cancer in a meta-analysis of observational studies. 2006 American society of clinical nutrition. Am J Clin Nutr May 2006 vol. 83 no. 5 1126-1134

13. Karthanasi et al. TGF $\beta 1$, Smad-2/3, Smad-1/-5/-8 \& Smad-4 signaling factors are expressed in ameloblastomas, adenomatoid odontogenic tumors, \& calcifying cystic tumors: an immune histologic study. J Oral Pathol \& medicine (2013) 42:415-423.

14. Lea-Ding et al, J oral pathol Med 43:335-343 2013 John Wiley and sons A/S published by John Wiley and sons Ltd.

15. Stevens RG et al body iron stores and risk of cancer. N Engl J Med 1988; 319:1047-1055.

16. Okinawa $M$ et al. Assessment of protein expression \& gene status of human epidermal growth factor receptor (HER) family molecules in ameloblastomas. J Oral Pathol \& medicine (2013) 42, 5:424-434.

17. Oral cancer, $4^{\text {th }}$ ed. Sol Silverman, Publisher. B. C. decker Inc; p18-19.

18. Oral cancer, $4^{\text {th }}$ ed. Sol Silverman, Publisher. B. C. decker Inc; $p$ xv.

19. Yang CZ et al. Elevated levels of serum growth differentiation factor 15 is associated with oral leukoplakia \& oral squamous cell carcinoma. J Oral Pathol (2014) 43:14-19.

\section{AUTHORS:}

1. Anitha $S$.

PARTICULARS OF CONTRIBUTORS:

1. Reader, Department of Periodontics, JSSDCH, JSSU.

FINANCIAL OR OTHER COMPETING INTERESTS: None
NAME ADDRESS EMAIL ID OF THE CORRESPONDING AUTHOR:

Dr. Anitha S,

Reader, Department of Periodontics, JSSDCH, JSSU.

E-mail: anithasu79@gmail.com

Date of Submission: 17/06/2015.

Date of Peer Review: 18/06/2015.

Date of Acceptance: 01/07/2015.

Date of Publishing: 08/07/2015. 